(e-migrinter)

\section{e-Migrinter}

$13 \mid 2015$

Mobilités, circulation migratoire et transnationalisme au prisme des réflexions méthodologiques de jeunes chercheur(e)s en sciences sociales

\title{
L'approche « réseaux » dans les études migratoires
}

Un cas d'étude les trajectoires scolaires des étudiants maliens en France et au Maroc

\section{Niandou Touré}

\section{OpenEdition}

\section{Journals}

Édition électronique

URL : https://journals.openedition.org/e-migrinter/549

DOI : 10.4000/e-migrinter.549

ISSN : 1961-9685

Éditeur

UMR 7301 - Migrinter

Référence électronique

Niandou Touré, «L'approche « réseaux » dans les études migratoires », e-Migrinter [En ligne], 13 | 2015,

mis en ligne le, consulté le 20 mai 2021. URL : http://journals.openedition.org/e-migrinter/549 ; DOI : https://doi.org/10.4000/e-migrinter.549

Ce document a été généré automatiquement le 20 mai 2021.

Tous droits réservés 


\section{L'approche « réseaux » dans les études migratoires}

Un cas d'étude les trajectoires scolaires des étudiants maliens en France et au Maroc

Niandou Touré

\section{Introduction}

1 Les approches explicatives des migrations peuvent être regroupées en trois grandes catégories qui correspondent aux trois principales échelles d'analyse. La perspective micro-individuelle, d'inspiration néoclassique, postule la rationalité du migrant, perçu comme acteur de son déplacement qui résulte d'un calcul en termes de coûts-avantages (Sjaastad, 1962 ; Lee, 1966). La deuxième catégorie d'analyse est l'approche systémique des migrations, ces dernières sont alors analysées à l'échelle macroscopique afin d'expliquer les structures migratoires, des flux notamment (Burawoy, 1976). L'approche «réseaux » apparaît comme la troisième échelle d'analyse. Intermédiaire entre les deux premières, elle circonscrit l'analyse à l'échelle méso des unités sociales telles que la famille, les associations, etc. Le sociologue Thomas Faist précise que cet angle d'analyse n'occulte pas pour autant le caractère hétérogène des projets et des ambitions des acteurs sociaux qui peuvent même être antagonistes dans les cercles les plus nucléaires tels que la famille (Faist, 1997 : 98). Dans ce cadre d'analyse, l'accent est mis sur l'importance des relations sociales dans les différentes phases du projet migratoire, car en dépit du caractère individuel apparent de la migration, elle dépend aussi beaucoup de dynamiques sociales (Stark \& Bloom, 1985).

Chacun de ces cadres d'analyse recourt à une méthodologie de recherche qui lui est spécifique. Dans le cadre de nos travaux de recherche qui portent sur la mobilité pour études des Maliens au Maroc et en France, l'approche par les « réseaux » est apparue la plus pertinente sur le terrain. En effet, en raison de l'implication très forte des cercles familiaux dans le projet migratoire des étudiants, et parce que la circulation de l'information sur les établissements d'enseignement supérieurs à l'étranger se fait à 
travers différents intermédiaires, cette approche s'est avérée nécessaire au cours de notre travail de terrain.

Dans cet article, nous restituons la méthodologie de recherche adoptée dans le cadre de notre thèse de doctorat et expliquons la nécessité d'avoir une lecture d'ensemble de la mobilité pour études à la lumière des résultats de nos recherches de terrain. Une fois présentés les fondements théoriques de l'approche « réseaux » et ses implications sur la méthodologie de la recherche, nous expliquerons comment, au fil de nos différentes enquêtes, elle a permis de mieux nous renseigner sur les sentiers de la mobilité à travers les acteurs qui jouent, à côté des étudiants, un rôle plus ou moins déterminant : familles, amis, recruteurs des universités, politiques gouvernementales, etc. L'article se clora sur la façon dont le terrain et la théorie se nourrissent mutuellement tout au long de la recherche. Nous détaillerons alors comment l'approche "réseaux» a facilité l'identification des éléments comparés de notre recherche.

\section{L'approche « réseaux » en questions}

\section{Les « réseaux » ou la focale sur les acteurs intermédiaires de la migration}

4 S'inspirant des approches explicatives qui lui sont antérieures tout en s'y opposant, l'approche par les « réseaux » dans les études migratoires connaît son essor à partir des années 1980 dans la littérature anglo-saxonne. Les économistes Stark et Bloom (1985), puis les sociologues Boyd (1989) et Massey (1988, 1990), ou encore Faist (1997), démontrent, chacun à leur façon, que les réseaux sociaux sont des facteurs qui se situent à mi-chemin entre les structures migratoires et les décisions individuelles des migrants (Piché, 2013). La thèse de Thomas Faist, qui a schématisé l'influence des liens symboliques et du capital social, a beaucoup contribué à la construction de notre méthode de recherche qui met les acteurs intermédiaires de la migration au centre de l'analyse. La migration est dans ce cas prise comme un tout qu'on peut décomposer en plusieurs étapes, regroupant plusieurs acteurs. Et l'étude d'un phénomène de cette nature peut être facilitée par la perspective d'analyse longitudinale de la mobilité pour études.

5 La littérature scientifique sur les études migratoires a souligné l'importance d'une analyse simultanée dans les pôles de départ et d'arrivée de la migration. Cependant, à quelques exceptions près, il apparaît que cette analyse n'est pas nécessairement prise en compte dans les recherches sur la mobilité académique, qui est une configuration migratoire singulière. Dans les travaux de Gérard $(2003,2008)$ et Mazzella (2009), on retrouve des analyses des itinéraires scolaires et sociaux des étudiants - Marocains en France pour le premier, Mauritaniens en Tunisie pour la seconde - de façon diachronique. Cependant, assez souvent, les publications en la matière ont eu une portée statistique (Carrington, 1998) et mettent l'accent, soit sur la zone d'arrivée des étudiants (Coulon, Paivandi, 2003), soit sur les pays de départ à partir desquels on essaie de saisir les raisons de la "fuite » des étudiants (Kritz, 2013). Les travaux qui se sont intéressés à la compréhension des différentes phases de la mobilité estudiantine et à l'importance du capital social sont donc peu nombreux. Souvent, l'attention est davantage portée sur les pays de destination que sur les pays de départ des étudiants internationaux. 


\section{Les implications méthodologiques de l'approche " réseaux » : échelle méso et enquête multi-acteurs}

6 Au fil des entretiens, nous avons opté pour une démarche multi-acteurs et multi-sites, qui a consisté à interroger des étudiants et leur entourage, dans leur pays de formation (la France ou le Maroc) et dans le pays de départ (le Mali). Certes, la formation à l'étranger représente un investissement en termes de capital humain. Sa réalisation repose, en revanche, sur la capacité à mobiliser un ensemble de ressources sociales au sein desquelles la famille et les réseaux d'interconnaissance occupent une place déterminante. Il ressort de notre recherche que ce capital social est particulièrement essentiel dans les différentes phases du projet d'études à l'étranger. C'est ce capital qui légitime l'intérêt méthodologique de mettre l'étudiant au centre d'une analyse d'ensemble qui permet de comprendre qu'à l'origine de la mobilité pour études, il y a une stratégie dans laquelle les réseaux familiaux et amicaux jouent un rôle clé.

7 Les trajectoires étudiantes sont ainsi analysées de sorte que l'étudiant devient l'acteur central et dont la migration s'effectue dans le sillage d'autres acteurs et facteurs sociologiquement importants: ses amis, sa famille, son histoire scolaire, etc. La compréhension de cette mobilité pour études requiert alors la prise en considération de ce large spectre d'action. Ce postulat théorique est à l'origine de notre méthodologie qui repose sur un travail auprès des différents acteurs de la mobilité, aussi bien dans le pays d'émigration que dans celui d'immigration.

8 Deux postulats ont guidé notre choix méthodologique. D'une part, la mobilité pour études, même si elle se distingue des autres formes migratoires professionnelles ou familiales, reste néanmoins une migration internationale. D'autre part, les catégories socioprofessionnelles des parents, l'autre aspect de notre recherche, pose beaucoup de difficultés d'analyse tant la nomenclature des professions en Afrique est complexe (Darbon, 2011). L'approche par les réseaux permet non seulement de poser le regard sur les différents espaces de la mobilité étudiante, du Mali vers le Maroc et du Mali vers la France, mais aussi et surtout de s'intéresser aux réseaux et aux acteurs intervenant dans la réalisation du projet de scolarité à l'étranger.

9 Ainsi, face à la double nécessité de penser la mobilité étudiante des Maliens en France et au Maroc, et afin de saisir le caractère multidimensionnel des catégories socioprofessionnelles (CSP) de leurs parents, nous avons fait le choix d'une recherche multi-située et multi-acteurs. Au cours des deux premières années du doctorat, nous avons ainsi réalisé trente-cinq entretiens semi-directifs auprès des étudiants maliens $\mathrm{du}$ Maroc et vingt-sept auprès de leurs compatriotes étudiant en France. L'échantillonnage a été réalisé avec l'aide des associations d'étudiants maliens de France et du Maroc, avec un travail de diversification des profils des étudiants en ce qui concerne la discipline d'étude, le genre, la CSP ou la durée du séjour à l'étranger. Le but de cette démarche était de comprendre les procédés de mobilisation des ressources sociales de la mobilité. Celle-ci requiert nécessairement un fort capital économique (Touré, 2014) ${ }^{1}$, un capital culturel symbolisé par le diplôme du baccalauréat au minimum et, dans une mesure variable, un capital social fait de toutes les relations familiales et amicales des étudiants.

$10 \mathrm{Au}$ Maroc, nous avons travaillé dans les villes de Rabat, Casablanca et Fès. En France, ce travail a été réalisé principalement à Paris, mais également à Grenoble. Ces différentes 
villes ont été choisies parce qu'elles présentent, chacune, un intérêt particulier pour l'analyse sociologique de la mobilité étudiante. Rabat est la ville des plus grandes facultés marocaines de médecine et de pharmacie, à côté de Casablanca qui abrite le tiers des écoles supérieures privées reconnues par le ministère marocain de l'enseignement supérieur ${ }^{2}$. À Fès, il est possible d'observer les tendances actuelles de la mobilité étudiante dans le secteur privé où certaines écoles accueillent en majorité des étudiants subsahariens, nouveaux visages de la présence étrangère au Maroc (Laouali, Meyer, 2012)3. En France, Paris est au cœur de la distribution géographique des structures d'enseignement. À elle seule, la région Île-de-France accueille dix-sept universités ainsi que la plupart des grandes écoles les plus réputées. Quant à la ville universitaire de Grenoble, elle offre l'opportunité de voir comment la présence institutionnalisée des étudiants venus dans le cadre de la coopération bilatérale a entrainé une concentration importante d'étudiants maliens dans la région ${ }^{4}$. En outre, le choix d'une ville de province de taille moyenne permet de ne pas éluder un aspect déjà très étudié des stratégies de mobilité des étudiants internationaux qui choisissent les petites villes pour leur coût de la vie moins élevé et une adaptation espérée plus souple à leur nouvelle vie (Lanoue, 2008).

11 Les entretiens réalisés auprès des étudiants, mais également de quelques diplômés de ces différentes villes nous ont ensuite conduit au Mali, dans les villes d'origine de certains étudiants ayant accepté que nous allions à la rencontre de leurs parents. Pour la majorité d'entre eux, les parents vivent à Bamako, la capitale du pays. Nos entretiens se sont déroulés sous la forme de récits de vie où une importance particulière a été accordée aux itinéraires scolaires et migratoires de l'étudiant et de sa famille. L'une de nos principales hypothèses était que la propension à étudier à l'étranger est liée au caractère plus ou moins "institué » de l'école et de la formation à l'étranger dans la famille, soit grâce au parcours d'un des deux parents, d'un membre de la fratrie, ou même d'un oncle ou d'une tante. Nous postulions aussi que les aptitudes académiques individuelles de l'étudiant pèseraient davantage que le capital économique des parents dans la probabilité de faire des études supérieures hors du Mali.

C'est ainsi que nous avons réalisé des entretiens qui incluaient des questions sur les niveaux de formation des parents, sur leurs professions et sur l'influence qu'ils ont eue dans les différentes phases du projet d'études à l'étranger de leurs enfants. Nous avons ensuite jugé utile de compléter notre corpus avec d'autres entretiens auprès des parents au Mali, notamment afin de mieux comprendre la catégorie sociale en croisant les discours et représentations des étudiants avec ceux de leurs parents. Dans certains cas, nous avons aussi fait des entretiens auprès d'un membre de la fratrie de l'étudiant lorsque celui-ci est dans le même pays de formation ou dans un de nos terrains d'enquête. Pour cinq des étudiants enquêtés, nous avons pu réaliser des interviews avec les frères/sœurs étudiant dans le même pays (deux d'entre eux) ou dans un autre pays (trois d'entre eux). En plus de la famille des étudiants, des entretiens ont été réalisés auprès des institutions en charge des mobilités, ainsi qu'auprès des représentants de certaines écoles à l'étranger. Dans ce large éventail d'interviews, il est apparu que la famille restait l'acteur le plus important de la mobilité pour études. 


\section{Les voies de la mobilité académique à partir du Mali}

\section{Capital culturel et mobilité pour études : le rôle de la formation supérieure à l'étranger des parents}

Nous postulions, au début de notre recherche, que le capital culturel, et dans une moindre mesure, le capital social, devaient jouer un rôle déterminant dans la probabilité d'étudier à l'étranger. Cette hypothèse est inspirée de travaux antérieurs. Qu'il s'agisse des Mauritaniens étudiant les sciences de la médecine en Tunisie (Mazzella, 2009), des étudiants des grandes écoles françaises (Bourdieu, 1989) ou des Marocains en France (Gérard, Proteau, 2008), l'éducation des parents ou leur familiarité avec les codes de l'école, jouent un rôle important dans les trajectoires scolaires des générations suivantes. Cette tendance est confirmée auprès des étudiants maliens aussi bien au Maroc qu'en France. Il apparait que les parents des étudiants maliens inscrits dans l'enseignement supérieur privé du Maroc sont globalement plus instruits que ceux du groupe des étudiants boursiers, aux niveaux très hétérogènes. Les parents dotés d'un plus grand capital culturel s'impliquent plus - aussi bien financièrement qu'intellectuellement - dans la définition ou la réorientation des trajectoires scolaires de leurs enfants.

Encadré $\mathrm{n}^{\circ} 1$ : Capital scolaire et mobilité étudiante : le parcours de $\mathrm{R}$, étudiante malienne à Rabat.

R., née en 1988 de deux parents enseignants l'un dans le secondaire (mère) et l'autre dans le supérieur (père) au Mali, est titulaire d'un diplôme d'ingénieur obtenu à Sup'Telecom Rabat. En 2012, elle préparait un master en Finance et Stratégie dans une autre école privée de Rabat. Elle est issue d'une famille imprégnée de la culture de la mobilité. Son père a effectué une partie de ses études supérieures en Algérie. Les huit frères et sœurs de R. ont étudié en Chine, au Ghana, en France et aux États-Unis, où l'un d'eux s'est établi. Elle explique avoir prospecté les écoles tunisiennes et marocaines à défaut d'avoir obtenu un visa pour la France. Le choix du Maroc par rapport à la Tunisie, d'après elle, relève du hasard, mais reste néanmoins un meilleur choix que de faire sa formation universitaire au Mali. Habituée des écoles privées dans lesquelles elle a fait toute sa scolarité primaire et secondaire, elle espère multiplier ses chances d'accès à l'emploi dans son pays d'origine grâce à ce passage à l'étranger qu'elle estime «naturel».

\section{L'expérience migratoire du réseau familial, une ressource peu mobilisée par les étudiants}

Si l'on observe les histoires de vie des étudiants maliens du Maroc, l'histoire migratoire semble avoir un impact limité sur les trajectoires scolaires, et ce, même lorsque le motif de la migration des parents avait été académique. Sur l'ensemble de notre échantillon, seule sept personnes ont déclaré que leurs parents avaient effectué des études à l'étranger. Et pour la majorité d'entre elles, ces parents ont étudié en ex-URSS. Quand leurs parents n'ont pas eux-mêmes eu d'expérience migratoire, les étudiants rapportent les exemples d'oncles ou de tantes ayant reçu une formation à l'étranger. En 
France, cependant, les familles installées depuis longtemps sur place, ont servi de relais fiables pour certains étudiants, particulièrement les étudiantes confiées par leurs parents des membres proches de la famille. Mais ces relais sont toujours ponctuels, les étudiants sont hébergés le temps qu'ils deviennent autonomes en trouvant un travail à temps partiel et un logement ${ }^{5}$. Le caractère institué de l'école dans les histoires familiales a beaucoup plus d'influence sur la propension à étudier à l'étranger que les expériences migratoires des parents et des proches.

Cette importance accordée à l'institution scolaire, et en particulier aux études à l'étranger, se confirme dans l'analyse des entretiens que nous avons réalisés auprès de la famille des interviewés. En effet, les informations recueillies auprès des frères et sœurs rencontrés dans le même pays ou dans un pays de formation différent, nous apprennent l'existence d'une forme de conscience de classe. Ce qu'on pourrait comprendre comme la confirmation du rôle du capital symbolique du diplôme dans la légitimation des élites. Cette culture de l'école, là où elle était présente, se manifeste sous la forme d'un investissement sur le long terme des parents dans les parcours scolaires de leurs enfants.

Cependant, de part et d'autre de la Méditerranée, au Maroc comme en France, il apparaît que les négociations entre les familles maliennes et l'école - cet objet encore étranger pour la beaucoup d'entre elles ${ }^{6}$ - se poursuivent à mesure que les enfants gravissent les échelles de l'enseignement supérieur. Pour certains étudiants, premiers de leurs familles à faire des études supérieures, les attentes face à l'école sont encore plus grandes. Ces "porteurs d'étendards » ont été décrits dans des travaux antérieurs sur les étudiants internationaux (Gérard, Proteau, 2008). Ceux parmi les étudiants marocains de France que les sociologues Etienne Gérard et Laurence Proteau nomment "avant-gardistes», ont leurs homologues Maliens en France et au Maroc. Cette catégorie regroupe des parcours de scolarisation atypiques qui sont le fruit des politiques éducatives de masse encouragées dans les pays en voie de développement à partir de $1990^{7}$. Ce sont surtout des "transfuges », dont l'improbable réussite scolaire doit souvent à une rencontre avec un inspirateur qui peut être un professeur, un parent éloigné ou une figure publique de la réussite par l'école. S'ils sont de plus en plus nombreux à se former à l'étranger (Sy, 2011), ils sont davantage présents dans les catégories de boursiers que dans les catégories d'étudiants dits « individuels », c'est-àdire ceux qui sont les propres initiateurs de leur démarche de formation à l'étranger ${ }^{8}$. Il y a peu d'étudiants " pionniers " parce que les ressources de la mobilité pour études capital social, économique et culturel - sont concentrées entre les mains des catégories sociales les plus aisées.

Une observation des différents acteurs intervenant dans la chaîne de transmission de l'information sur la formation supérieure à l'étranger permet de comprendre la multiplicité des acteurs intermédiaires dont le rôle mérite d'être décrypté.

\section{Les « intermédiaires », des acteurs à part entière de la mobilité étudiante}

18 Jusque-là, nous avons distingué deux modalités de mobilité internationale pour études : les étudiants boursiers des coopérations bilatérales interétatiques ou des institutions internationales et les étudiants qui financent eux-mêmes leurs études à l'étranger. Cette deuxième catégorie représente plus de $95 \%$ des étudiants maliens en France ${ }^{9}$. Au 
Maroc également, les étudiants maliens non-boursiers sont plus nombreux que les boursiers, même s'il existe peu de données statistiques fiables sur les étudiants étrangers dans le royaume chérifien ${ }^{10}$.

En ce qui concerne les étudiants boursiers, l'information relative aux concours de sélection est relayée, normalement, par les médias nationaux, la presse et les sites internet dédiés. C'est pour cela que nous choisissons, ici, de nous intéresser à la circulation de l'information dans le cas de la mobilité des étudiants qui s'autofinancent. La diffusion de l'information n'est jamais parfaite. C'est là l'une des limites du modèle d'analyse néoclassique des migrations, qui fait du migrant un acteur rationnel opérant des choix qu'il juge optimaux. Dans le cas des étudiants maliens en mobilité, l'information - sur les écoles, les frais de scolarité, les conditions d'accès - est concentrée au sein de cercles sociaux restreints. Par conséquent, sa circulation dépend des intermédiaires qui en sont les porteurs. Selon qu'il s'agisse de la France ou du Maroc, cette information est propagée dans des canaux différents. Cette dissimilitude s'explique en partie par les différences entre les systèmes éducatifs des deux pays et entre leurs modalités d'accès aux études supérieures.

21 En France, l'entrée des étudiants étrangers se fait sous l'égide des institutions administratives qui ont été créées à cet effet. Depuis 2006, Campus France centralise toutes les demandes des candidats étrangers aux études supérieures en France. Ainsi, l'étudiant malien qui souhaite y étudier doit obligatoirement respecter une procédure de demande d'inscription, suivie d'une demande de visa et de la justification des ressources financières nécessaires pour séjourner en France. Pour presque tous les étudiants avec lesquels nous nous sommes entretenus en France, ces informations ont été obtenues auprès de leurs proches, de leurs amis ou de membres de leurs familles déjà étudiants en France. C'est le cas de H. qui explique qu'il a reçu l'aide de [son] cousin qui étudiait à Reims, et qui s'est quasiment occupé de toute la procédure de demande d'inscription ${ }^{11}$.

$22 \mathrm{Au}$ Maroc, les conditions d'accès à l'enseignement supérieur sont différentes. Les établissements publics accueillent uniquement les étudiants boursiers alors que les écoles privées accueillent les non-boursiers. Dans un tel schéma, la présence des étudiants étrangers dépend fortement des moyens de communication utilisés par les écoles privées dont l'essor a été promu par le gouvernement du Maroc à partir de 2000, avec la loi $01.00^{12}$ qui souligne la complémentarité entre les secteurs public et privé de l'enseignement supérieur. Ce qui devait être un remède aux maux de l'école marocaine (augmentation de la demande d'éducation due à la poussée démographique et insuffisance de la plaquette pédagogique) a vite trouvé écho dans les aspirations des catégories aisées de l'Afrique subsaharienne francophone.

Consciente de cette nouvelle donne, plusieurs écoles privées, dont certaines accueillent davantage d'étudiants subsahariens que de Marocains (Mazzella, 2009) adoptent des éléments de communication tournés vers les pays de l'Ouest africain. Outre une communication sur internet dédiée aux étudiants originaires du Gabon, de la Côte d'Ivoire, du Sénégal, du Mali, de la Guinée, etc., certains établissements désignent des représentants officiels ayant pour mission de recruter des étudiants dans leur pays d'origine. C'est la figure de l'étudiant-recruteur, qui se situe socialement dans la même catégorie que les étudiants qui peuvent s'acquitter des frais d'inscription des écoles privées (entre 2000 et 6000 euros par an). Intermédiaire et facilitateur, la description de son action permet de comprendre l'effet de la contingence et de la circulation de 
l'information sur les choix de mobilité pour études des Maliens. En effet, il recrute autour de lui des étudiants qui disposent des moyens financiers pour se former à l'étranger mais qui n'ont pu partir soit après échec de leurs tentatives, soit par méconnaissance de cette possibilité de formation à l'étranger.

Encadré $\mathrm{n}^{\circ} 2$ : Portrait de S.-M, étudiant et recruteur malien pour une école privée de Fès.

S.-M. a vingt ans et est étudiant en deuxième année du cycle Informatique et gestion à EPSIEL (École Polyvalente Supérieure d'Informatique et d'Électronique). L'école lui a été recommandée par une amie. Sans en apprendre davantage, il s'y est inscrit et a réussi à se faire désigner comme recruteur, après avoir proposé ses services à la direction de l'école, dès la fin de sa première année. Contre une rémunération qui varie entre 100 et 400 euros pour chaque étudiant recruté, le jeune homme s'active particulièrement sur les réseaux sociaux du net (Facebook, Twitter) pour diffuser l'information sur l'offre scolaire d'EPSIEL. Les deux dizaines d'étudiants qu'il a réussi à faire adhérer à l'école au titre de l'année universitaire 2013-2014 lui permettront d'alléger ses coûts de scolarité, estimés à 2.500 euros, et de grossir les rangs des étudiants maliens qui représentent déjà un quart des effectifs d'EPSIEL à Fès.

\section{Dynamique de recherche entre le terrain, la théorie et l'exploitation des données}

\section{Les voies de circulation de l'information : un monde d'intermédiaires}

À l'image des autres formes de migrations internationales, la mobilité étudiante n'est jamais un projet totalement individuel. Les capitaux socioéconomique et culturel sont des variables déterminantes dans la probabilité d'accomplir le projet de mobilité, ce que confirme l'importance des réseaux sociaux mobilisés dans les différentes phases du projet. Que nous parlions des étudiants boursiers ou des non boursiers, les sentiers qui préparent à une scolarité extérieure dépendent beaucoup de l'environnement social des individus. Dans les pays où la qualité de l'éducation supérieure est décriée (Diakité, 2000), la formation à l'étranger apparaît comme une aubaine dont tout le monde veut bénéficier. C'est pour cela que la mobilité est très souvent un projet encouragé, voire conçu en collaboration avec les parents. Dans le cas du Mali, c'est bien souvent le père qui participe à l'élaboration du projet d'études. La formation à l'étranger est donc organisée dans le pays de départ afin d'acquérir la plus-value du diplôme étranger. Cette plus-value est censée faciliter l'insertion « ici » et/ou «là-bas ». La formation en Tunisie ou au Maroc peut être fortuite, elle se fait au gré des rencontres personnelles de l'étudiant ou de l'un de ses parents. L'étudiant est donc en cela le premier acteur de sa mobilité. C'est lui qui effectue le voyage et qui s'enrichit de la formation à l'étranger, qui accroît son capital symbolique par l'acquisition d'un titre universitaire valorisé dans son pays de départ. Cependant, la mobilisation du capital social et économique de sa famille influe énormément sur la concrétisation de son projet.

In fine, il n'existe pas d'étudiant individuel. Sont désignés sous ce vocable d' "individuels", ceux qui ne disposent pas de financement d'institutions publiques ou privées. Ils sont initiateurs, avec leurs parents, de leur mobilité étudiante. 
Il y a donc un véritable paradoxe à les nommer comme tels parce que leur migration est plus collective qu'individuelle. Les risques et les espoirs d'une distinction ou d'une reproduction sociale sont alors partagés avec beaucoup d'acteurs, au premier rang desquels la famille.

Encadré $\mathrm{n}^{\circ} 3$ : Portrait d'un étudiant malien en France rejoint plus tard par ses frères et sœurs étudiants

O. est étudiant et termine son master 2 en Finance à Paris lorsque nous le rencontrons en juin 2012. Auparavant, il avait fait sa licence d'économie à Lyon. C'est aussi dans cette ville que ses deux sœurs et son frère font encore leurs études. 0 ., aîné de sa famille, a beaucoup contribué à la venue de ses cadets en France, qu'il a informés et préparés chaque fois que l'un d'eux réussissait son examen du baccalauréat. Son père, M. B., que nous avons rencontré en août 2012, est cadre dans une institution spécialisée de l'ONU. Dans le cas de la famille d'O., l'information était à disposition de son père qui a fait lui-même toutes les démarches pour scolariser son premier en France. 0 . a ensuite pris le relais et a transmis l'information à ses jeunes frère et sœurs. Les intermédiaires dans ce cas sont les membres de la famille proche, et confirment le rôle primordial des réseaux dans l'accomplissement des projets de mobilité académique.

\section{L'approche « réseaux » et la comparaison internationale}

Nous avons constaté la difficulté de comparer les attitudes individuelles des étudiants maliens en mobilité d'un pays à l'autre, parce que les ressorts de leur migration dépendent de logiques structurelles ou individuelles très éloignées. L'approche par les réseaux sociaux et l'échelle d'analyse méso ont alors facilité l'exercice de comparaison entre nos différentes destinations d'étude. Elles ont permis de voir quels étaient les mécanismes à l'œuvre dans les parcours des étudiants qui vont, d'une part, du Mali vers la France, et du Mali vers le Maroc, d'autre part. Nous avons construit des outils de comparaison à partir des observations de terrain. L'implication des réseaux familiaux ou amicaux, les relais de l'information sur les écoles et les bourses d'étude, le poids cumulatif de l'installation des étudiants dans une même ville, ou le mimétisme dans les orientations scolaires, ont été choisis entre autres points de comparaison dans notre analyse des trajectoires scolaires. La définition de ces outils de comparaison permet de mieux comprendre les spécificités de chacun de nos deux schémas de mobilité internationale des étudiants.

De façon plus spécifique, nous nous sommes intéressés aux personnes ressources désignées par les étudiants eux-mêmes dans leurs récits biographiques comme importantes dans leurs trajectoires scolaires et sociales. Nous avons ainsi décelé trois catégories clés de leurs réseaux sociaux, correspondant aux relais principaux de leur parcours migratoire. Il s'agit des personnes qui les ont soit inspirés soit guidés dans leur parcours académique au Mali et/ou à l'étranger, des parents ou amis qui ont rempli une mission d'informateur à propos des écoles ou des systèmes de bourse au Mali et/ou à l'étranger, et des personnes ressources qui leur ont apporté une contribution morale ou financière dès le Mali ou une fois arrivés au Maroc ou en France. 
Les ressources sociales mobilisées par les étudiants maliens en France et au Maroc ne sont pas les mêmes. En raison d'une histoire migratoire différente, les étudiants maliens de France s'appuient couramment sur des membres de leurs familles proches ou lointaines établies quelques fois depuis trois ou quatre décennies en Île de France. $\mathrm{Au}$ Maroc, où la formation supérieure des Maliens depuis les années 1960 n'a pas débouché sur une immigration durable, les relais locaux des étudiants maliens aujourd'hui sont avant tout d'autres étudiants ou jeunes diplômés. Ces relais ont un impact plus ou moins important sur les trajectoires scolaires des étudiants. Il ressort de notre analyse que le rôle joué par l'étudiant recruteur au Maroc dans les choix de formation des étudiants maliens est de rencontrer, informer et orienter, il est analogue à la fonction remplie par la figure de l'intermédiaire qui conseille les lycéens de terminale à Bamako dans leurs démarches administratives auprès de Campus France, ou même de l'hôte installé en région parisienne. Ces trois figures ont toutes trois, certes de façon distincte, une influence notable sur les trajectoires scolaires des étudiants. Les comparer, c'est aussi décortiquer la place des réseaux sociaux dans la mobilité pour études de régions a priori différentes.

\section{Conclusion}

La multi-dimensionnalité, consubstantielle au phénomène migratoire, requiert une analyse approfondie dans les espaces d'émigration et d'immigration. La mobilité pour études des Maliens au Maroc et en France n'échappe pas à cette exigence: ses déterminants sont à comprendre aussi bien au Mali que dans les pays de formation. La continuité entre ces différents espaces est assurée par des acteurs intermédiaires qui véhiculent l'information. L'approche par les réseaux sociaux permet de mieux comprendre les modalités de mobilité des étudiants en France et au Maroc. Nous avons démontré que parmi les multiples acteurs de la mobilité étudiante, les familles sont au cœur des stratégies déployées par les étudiants. Afin de situer plus clairement leur participation dans l'élaboration du projet migratoire, les entretiens réalisés avec les acteurs intermédiaires ont permis de mieux analyser un phénomène, la migration en général, qui est toujours à remettre dans le cadre de relations interpersonnelles qui en définissent les contours et les orientations. Nous avons ensuite appréhendé les autres aspects de cette recherche, tels que les catégories sociales d'origine des parents et les processus d'ascension, de reproduction ou de distinction par des analyses longitudinales aussi bien dans l'espace migratoire que dans le champ des relations sociales des étudiants, noyaux d'une mobilité qui mobilise différents acteurs.

Les approches systémiques et individuelles des migrations internationales ne suffisent pas toujours à expliquer les différentes formes de mobilité. L'échelle macroscopique rend compte des flux entre pays, l'échelle micro nous renseigne sur les déterminants de la décision individuelle de migrer, mais les interactions sociales, les dynamiques relationnelles, l'importance du capital social et ses implications en termes d'obligation envers les siens, ne peuvent s'entendre de façon idoine que dans l'optique d'une approche des migrations internationales par les réseaux sociaux. À l'ère de la reconfiguration des flux migratoires dont les destinations se diversifient, la recherche sur les mobilités des étudiants doit davantage varier les échelles d'analyse pour mieux saisir la complexité des phénomènes de circulation des hommes. 


\section{BIBLIOGRAPHIE}

Bourdieu, Pierre (1989) Noblesse d'État : Grandes écoles et esprit de corps, Paris, Éditions de minuit, 576 p. (Le sens commun).

Boyd, Monica (1989) Family and personal networks in international migration: Recent developments and new agendas, International Migration Review, vol. 23, n 3, pp. 638-670.

Burawoy, Michael (1976) The function and reproduction of migrant labour: Comparative material from Southern Africa and the United States, American Journal of Sociology, vol. 82, $n^{\circ} 5$, pp. 1031-1042.

Carrington, William J.; Detagriache, Enrica (1998) How big is the brain drain? Working paper, International Monetary Fund, 27 p. [réf. du 29 juin 2014] [Disponible sur internet].

Coulon, Alain ; Paivandi, Saeed (2003) Les étudiants étrangers en France : l'état des savoirs, Rapport pour l'observatoire de la vie étudiante (OVE), Université Paris 8, Centre de Recherche sur l'Enseignement Supérieur, $52 \mathrm{p}$.

Darbon, Dominique (2011) Quelle(s) classe(s) moyenne(s) en Afrique ? Une revue de littérature, Document de travail $n^{\circ} 118$ de l'Agence Française de Développement (AFD), Paris, 64 p., [réf. du 29 juin 2014] [Disponible sur internet].

Diakité, Drissa (2000) La crise scolaire au Mali, Nordic Journal of African Studies, vol. 9, n 3, pp. 6-28.

Faist, Thomas (1997) The crucial meso-level, in Hamar, T.; et alii. (dir.) International migration, immobility and development: multidisciplinary perspectives, Oxford, Berg, pp. 187-218.

Gérard, Etienne ; Proteau, Laurence (2008) Les conditions sociales de la promotion universitaire : « héritiers », « pionniers » et étudiants « d'avant garde », in Étienne, G. (dir.) Mobilités étudiantes Sud-Nord: Trajectoires scolaires de Marocains en France et insertion professionnelle au Maroc, Paris, Publisud, pp. 121-187.

Gérard, Etienne (2003) Les trajectoires sociales et scolaires des étudiants marocains en France : discours et pratiques, Rapport pour le Service de Coopération et d'Action Culturelle (SCAC) de l'Ambassade de France à Rabat (Maroc), Paris-Rabat, 341 p.

Kritz, Mary (2013) International Student Mobility and Tertiary Education Capacity in Africa, International Migration, vol. 53, $\mathrm{n}^{\circ} 1$, pp. 29-49.

Lanoue, Eric (2008) Les conditions sociales de l'expérience migratoire : logement, ressources matérielles et emplois des étudiants marocains en France, in Gérard, É. (dir.) Mobilités étudiantes Sud-Nord: Trajectoires scolaires de Marocains en France et insertion professionnelle au Maroc, Paris, Publisud, pp. 61-119.

Laouali, Souleymane ; Meyer, Jean-Baptiste (2012) Le Maroc : pays d'accueil d'étudiants étrangers, Hommes \& Migrations, n 1300, pp. 114-123.

Lee, Everett (1966) A theory of migration, Demography, vol. 3, n 1, pp. 47-57.

Massey, Douglas (1988) Economic development and international migration in comparative perspective, Population and Development Review, vol. 14, n 3, pp. 383-413.

Massey, Douglas (1990) Social structure, household strategies, and the cumulative causation of migration, Population Index, vol. 56, $\mathrm{n}^{\circ}$ 1, pp. 3-26 
Mazzella, Sylvie (dir.) (2009) La mondialisation étudiante : le Maghreb entre Nord et Sud, Paris, Karthala, $404 \mathrm{p}$.

Piché, Victor (2013) Les théories migratoires contemporaines au prisme des textes fondateurs, Population, vol. 68, $\mathrm{n}^{\circ} 1$, pp. 153-178.

Sjaastad, Larry (1962) The costs and returns of human migration, Journal of Political Economy, vol. 70, n 5(2), pp. 80-93.

Stark, Oded; Bloom, David E. (1985) The new economics of labor migration, The American Economic Review, vol. 75, $\mathrm{n}^{\circ}$ 2, pp. 173-178.

Sy, Harouna (2011) Marché de l'enseignement supérieur Sud-Sud. Les carabins marocains à l'université de Dakar, in Leclerc-Olive, M. ; Scarfo Ghellab, G. ; Wagner, C. (dir.) Les mondes universitaires face au marché : circulation des savoirs et pratiques des acteurs, Paris, Karthala, pp. 93-106.

Touré, Niandou (2014) Les étudiants maliens dans l'enseignement supérieur privé au Maroc, Hommes et migrations, $\mathrm{n}^{\circ} 1307$, pp. 29-36.

\section{NOTES}

1. Dans nos travaux antérieurs, nous avons estimé les frais de scolarité dans les établissements d'enseignement supérieur privé du Maroc entre 2.200 et $5.500 €$ (Touré, 2014). Campus France, l'organisme public chargé de la gestion des candidatures des étudiants étrangers voulant étudier en France, exige de ces derniers des ressources financières mensuelles de $615 €$.

2. D'après la liste établie par le ministère marocain de l'enseignement supérieur et de la recherche scientifique, 230 établissements privés étaient autorisés au titre de l'année universitaire 2013-2014. Parmi ces 230 écoles et universités, 77 se trouvent à Casablanca.

3. D'après Meyer et Laouali, les étudiants étrangers au Maroc, qui sont en grande majorité originaires d'Afrique subsaharienne, ont vu leur nombre croître de façon continue pendant la décennie 2000-2010, passant ainsi de 3628 en 1998-1999 à 15577 en 2010-2011. Cette progression en nombre absolu et au ratio d'étudiants étrangers, doit beaucoup à l'essor du secteur privé depuis le début du nouveau millénaire.

4. Le programme " 300 cadres pour le Mali ", initié en 1999 entre les gouvernements du Mali et de la France, sélectionne, depuis 2000, 5 à 20 bacheliers maliens par an, sur concours, qui poursuivront leurs études supérieures à Valence, dont les établissements sont rattachés à l' université de Grenoble-Alpes. Autour de ce programme, s'est développé peu à peu un réseau d'étudiants maliens dont le nombre a considérablement crû en Rhône-Alpes en une dizaine d'années.

5. Par exemple, nous avons rencontré trois étudiants en Île de France, hébergés soit par une sœur de leur maman, soit une sœur de leur père ou un cousin. Cet hébergement a duré entre six mois et deux ans pour les trois étudiants que nous avons rencontrés.

6. Pour de plus amples développements sur le caractère exogène de l'École au Mali et en Afrique de l'Ouest, et sur les rapports entre familles et écoles, voir notamment les travaux de Jean-Yves Martin et de Marie-France Lange.

7. Cette année-là, s'est tenue la première conférence mondiale sur l'éducation pour tous, à Jomtien (Thaillande).

8. Cette appellation est couramment utilisée par les structures chargées de produire les informations statistiques sur les flux étudiants à partir des pays d'accueil. 
9. Au titre de l'année universitaire 2013-2014, il y avait 2285 étudiants maliens dans les universités françaises (DEPP - Ministère de l'éducation Nationale, 2014). Parmi eux, une centaine sont boursiers, soit moins de $5 \%$.

10. Sur la base de la liste électorale établie par l'ambassade du Mali au Maroc, nous estimions le nombre d'étudiants maliens au Maroc à 1800 en 2012 alors que l'Agence Marocaine de Coopération Internationale, l'AMCI, dénombrait autour de 500 étudiants maliens boursiers de la coopération.

11. Entretien réalisé le $07 / 01 / 2012$ à Grenoble au domicile de $\mathrm{H}$, alors étudiant en troisième année de licence Maths-Informatique à l'université Joseph Fourier.

12. Loi $n^{\circ} 01.00$ portant organisation de l'enseignement supérieur au Maroc [Disponible sur internet].

INDEX

Index géographique: Maroc, France

Mots-clés : étudiants, enseignement supérieur, mobilité géographique, projet migratoire, réseaux d'entraide, méthodologie, enquête, Maliens

\section{AUTEUR}

NIANDOU TOURÉ

Doctorant en sociologie, Université Paris Descartes - CEPED

toureniandou@gmail.com 